\title{
PROBLEM OF MEASURING THERMAL CONDUCTIVITY OF POLYURETHANE THERMAL INSULATION OF LIQUID HYDROGEN TANKS FOR LAUNCH VEHICLE IN OPERATIONAL ENVIRONMENT
}

\author{
Sergey Kravchenko ${ }^{1}$, Nikolaj Kuleshov ${ }^{1}$, Natalia Panova ${ }^{1}$, Ugis Cabulis ${ }^{2}$, \\ Romass Pauliks $^{3}$, Vladimir Shestakov ${ }^{1}$, Sergey Bratarchuk ${ }^{1}$ \\ ${ }^{1}$ SIA Cryogenic and vacuum systems, Latvia; ${ }^{2}$ Latvian State Institute of Wood Chemistry, Latvia; \\ ${ }^{3}$ Ventspils University of Applied Sciences, Latvia \\ sergey@cvsys.eu
}

\begin{abstract}
The problem of polyurethane cryogenic thermal insulation quality control has become more and more urgent. Such thermal insulation has a wide range of applications from space technology to medical technology. In comparison with traditional cryogenic thermal insulation, such as screen-vacuum, vacuum-zeolite, vacuum-perlite, and vacuum-fibre insulation, polyurethane thermal insulation has some undeniable advantages, such as low cost, high manufacturability and low labour intensity of the insulation of the structure, no need for periodic maintenance, high resistance to vibration, shock, etc. At the same time, full reproducibility of insulation properties is a problem. To guarantee successful use of the thermal insulation, especially in such critical areas as space industry applications, it is necessary to monitor its thermal conductivity in the range of operating temperatures as the main parameter that determines consumer properties. Measuring the thermal conductivity of materials is quite difficult, since thermal conductivity cannot be measured directly. It is even more difficult to measure thermal conductivity in space technology operating conditions - in a vacuum and low temperatures. SIA Cryogenic and vacuum systems, the Latvian State Institute of Wood Chemistry, and the Ventspils University of Applied Science, in the framework of the EDRF financed "Cryosiltmer" project are developing a low-temperature thermal conductivity measurement method and a test facility on its basis. It will measure the thermal conductivity of polyurethane thermal insulation in air pressure/vacuum or helium environment in the cold end temperature range of 10-30 K. The project development is quite complicated, because to obtain correct measurement of thermal conductivity, it needs to minimize the temperature field gradients in the measurement system in such temperature range. During the project implementation the measurement system thermal environment model was developed. The modelling results showed that it is impossible to use directly the method used in existing NASA facility due to the reasons of great thermal loses. Then, the modelling helped find the solution, which allows to use a market available cryogenic cooler to make the measuring facility - to design proper intermediate liquid nitrogen cryostat. Now the facility is under assembling and testing. Preliminary vacuum and cryogenic tests show proper functioning of the cryostat and its capability to high vacuum operation. The proposed approach, described in the paper can help solve the problem of the thermal conductivity measurement under liquid hydrogen temperatures. After confirmation of the thermal conductivity stability in the created test facility the polyurethane thermal insulation will be used in liquid hydrogen tanks of the ESA "Ariane-6" launch vehicle.
\end{abstract}

Keywords: cryogenics, polyurethane, thermal conductivity.

\section{Problem of the launch vehicle polyurethane cryogenic thermal insulation}

Meeting modern requirements to improve the efficiency and environmental friendliness in the design of new launch vehicles leads manufacturers to ever wider use of rocket engines, in which the propellant components are cryogenic fluids: liquid oxygen is an oxidizer and liquid hydrogen is a fuel. The reason is that this pair provides maximum efficiency with maximum specific impulse up to $477 \mathrm{~s}$, [1] Tab. 4.2.1-6, and as a product of their burning, water is formed. Launch vehicles, taking satellites from the Earth's surface to orbit, as a rule, have great capacity cryogenic propellant tanks, for example, the first stage of the Atlas V launch vehicle has about 287 tons of propellant [2].

The specially designed cryogenic insulated tanks are required to avoid large losses of cryogenic components, low-temperature launch vehicle and launching site structure destruction, and explosion hazards associated with cryogenic liquids boiling and their vapor leakage. At the same time, the design requirements of the rocket and space technology do not allow the use of the traditional types of cryogenic thermal insulation: screen-vacuum, perlite, and zeolite vacuum, and fiber-vacuum for a large mass of double-walled vacuum vessels and the high probability of their depressurization under flight overload conditions. The conventional thermal insulation (foams with open or closed cells or fibers) is unsuitable for such applications, due to physical and mechanical instability (brittleness, etc.) at cryogenic temperatures, high permeability to air gases and water vapor, the possibility of getting wet with liquid gases, condensed from the ambient air, the formation of ice and, accordingly, the destruction and loss of the thermal insulation properties. 
The only types of cryogenic insulation used so far in rocketry have been aerogel, perlite or zeolite powder bulk insulation. Their main disadvantages are low manufacturability, high labor intensity, and the need to create load-bearing and enclosing structures where insulation is poured, or a complex process of gluing aerogel mats and a protective enclosing structure, as well as high thermal conductivity at atmospheric pressure, e. g., the coefficient of thermal conductivity of aerogel mats at a pressure of $1 \cdot 10^{-}$ ${ }^{3}$ Torr is $1.83 \cdot 10^{-3} \mathrm{~W} \cdot \mathrm{m}^{-1} \cdot \mathrm{K}^{-1}$, and at atmospheric pressure $1.434 \cdot 10^{-2} \mathrm{~W} \cdot \mathrm{m}^{-1} \cdot \mathrm{K}^{-1}$, and for perlite powder these indicators are equal to $1 \cdot 10^{-3}$ and $3.5 \cdot 10^{-2} \mathrm{~W} \cdot \mathrm{m}^{-1} \cdot \mathrm{K}^{-1}$, respectively [3].

It should be noted that with an increase in the parking time of the launch vehicle fueled with cryogenic components outdoor at atmospheric pressure, the thermal conductivity of these types of insulation begins to increase significantly. This is due to the fact that as air penetrates into the thermal insulation material, cryoadsorption of water vapor, carbon dioxide, oxygen, and nitrogen from the air by the thermal insulation material occurs and the subsequent formation of solid particles and liquid droplets with high thermal conductivity in the gaps between the particles [4]. Since the launch vehicle can be at the launch position in a fueled state for up to 3 days, the thermal conductivity of the thermal insulation is constantly increasing and under unfavorable conditions can reach $1-1.5 \mathrm{~W} \cdot \mathrm{m}^{-1} \cdot \mathrm{K}^{-1}[5]$.

Technical solutions, based on the traditional thermal insulation, are especially dangerous for the storage of liquid hydrogen, taking into consideration, that hydrogen has the melting point of $13.99 \mathrm{~K}$, and its boiling point is $20.271 \mathrm{~K}$. In this regard, extensive research has been carried out in the EU space science and industry, and closed cells materials, such as polyurethane (PU) and polymethacrylimide (PMI) have been proposed as the main directions in the field of cryogenic thermal insulation [6] for design and development of new European space launch vehicles using liquid hydrogen as a fuel.

Work on the creation of cryogenic thermal insulation based on polyurethane has been carried out at the Latvian State Institute of Wood Chemistry (LSIWC) since the 1980s. The research results of this institute were used in the development of the Energia-Buran reusable launch vehicle (RLV). After Latvia joined the European Space Agency (ESA) Plan for European Cooperating States (PECS) program, ESA European Space Research and Technology Center (ESTEC) specialists, while creating the concept of the new Ariane-6 launch vehicle, based on the previous experience of LSIWC, made a decision to involve LSIWC to work on the project of creating technology for forming thermal insulation of tanks with liquid hydrogen, based on covering their shell with polyurethane foam.

On the recommendation of ESA ESTEC specialists, LSIWC turned to SIA "Cryogenic and vacuum systems" (CVS) to create a facility for testing thermal insulation in operational conditions. To create a measuring system for this facility, the parties invited a team of programmers and electronics engineers from the Ventspils University of Applied Sciences (VEA) to cooperate. To ensure funding for the project, an application was submitted to the European Regional Development Fund, as a result, from 01.11.2019 CVS, LSIWC and VEA are implementing the ERDF project No. 1.2.1.1/18/A/006 Research No. 2.8. "Cryogenic Insulation Thermal Conductivity Testing System".

\section{Problem of thermal conductivity measurement at the helium temperature range}

To ensure that PU thermal insulation is free of the above disadvantages and does not pose the abovedescribed hazards to the launch vehicle, caused by its thermal conductivity, is increasing the need to proof the stability of its thermophysical properties, first of all, stable thermal conductivity under operating conditions. Therefore, to verify the insulation of cryogenic tanks, and, accordingly, to ensure the reliability of launch vehicles, it is necessary to create a method and test facility that ensures the measurement of the thermal conductivity of polyurethane thermal insulation under operating conditions, that is, at a temperature of liquid hydrogen (about $20 \mathrm{~K}$ ), under conditions of pressure change from atmospheric to high vacuum and in presence of helium diffusion.

The main purpose of the developed research facility is to determine the thermal conductivity of cryogenic thermal insulation made of polyurethane foam (PUF) in the temperature range of liquid hydrogen (14-20 K), including under conditions of helium saturation of the thermal insulation. This last condition corresponds to the situation occurring in real flight preparation conditions, when helium penetrates into the thermal insulation through the tank shell joints when the tank is pressurized with helium, which is necessary to supply hydrogen to the rocket engine turbopump unit. 
To ensure the relevant environment condition simulation, as described in [7], it is necessary to cool the test specimen and the thermal conductivity measuring instrument to the temperature range of liquid hydrogen and to remove the excess thermal power released during the measurement from the measuring zone. Also, it needs to make the measurement zone hermetically sealed, to provide the possibility to fill the internal volume of the measuring zone with helium and at the same time to ensure its effective vacuum thermal insulation from the external heat flux.

Such cryogenic cooling challenge can be solved only by creating a high-vacuum cryostat with effective thermal insulation [8], move inside it a sealed pressure vessel with thermal conductivity measuring instrument, and cooling it using multistage pulse tube, Stirling or Gifford-McMahon helium cryocoolers [9].

\section{An approach to solving the problem of thermal conductivity measuring at helium temperature range and the need to create a methodology}

To solve the design problems, first of all, a search and analysis of standards for determining thermal conductivity and methods adopted in the space industry that are acceptable for such an application (low thermal conductivity, elastic material, low temperature) were carried out, these documents [10-13] were analyzed and taken as a basis for the development the design measurement scheme and the measuring device itself. The above-mentioned sources suggest the following principle for measuring the specimen thermal conductivity. A material specimen in the form of a flat plate of the circular or rectangular shape of known area and thickness is placed between two plates, on which a temperature field is created that has a high uniformity (minimum temperature gradient). In this case, one of the plates is cooled to the required measurement temperature. The second plate contains an electric heater, the electrical power supplied to which is precisely controlled. The temperatures of the hot and cold sides of the specimen are also measured. Then the thermal conductivity is defined as:

$$
\lambda=\frac{\Phi d}{A\left(T_{1}-T_{2}\right)},
$$

where $\lambda$-thermal conductivity, $\mathrm{W} \cdot \mathrm{m}^{-1} \cdot \mathrm{K}^{-1}$;

$\Phi$ - average power supplied to the metering section of the heating unit, $\mathrm{W}$;

$d$ - average specimen thickness, $\mathrm{m}$;

$A$ - metering area, $\mathrm{m}^{2}$;

$T_{1}$ - average specimen hot side temperature, $\mathrm{K}$;

$T_{2}$ - average specimen cold side temperature, $\mathrm{K}$.

In accordance with the recommendations of ESTEC experts, an experimental facility created in 2011 at the Florida State University in the interests of the NASA Kennedy Space Center and the Florida Center for Advanced Aero-Propulsion (FCAAP), was taken as the basis of the project for the technical implementation of the specified principle of thermal conductivity measuring [14].

A diagram of the Florida State University experimental facility (FSU facility) is shown in Fig.1.

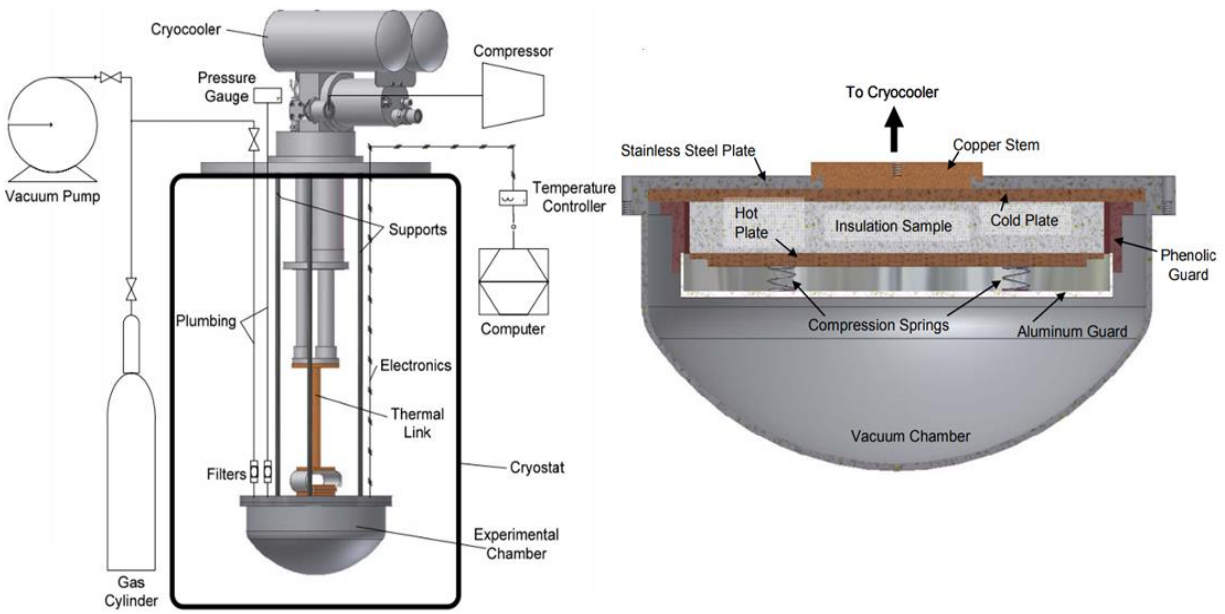

Fig. 1. Diagram of the Florida State University thermal conductivity measuring facility [11] 
A thermophysical modeling of the FSU facility was carried out by the authors. During the modeling, the following was accepted. In accordance with the requirements of $[10 ; 12 ; 13]$, the minimum diameter of the test specimen is $300 \mathrm{~mm}$. When redesigning the facility proposed in [14], taking into account the diameter of the test specimen required by the standards, a constructive model has been created and the geometric dimensions of the experimental chamber (name from Fig. 1) and cryostat were calculated. Fragment of the first developed design model is shown in Fig. 2.

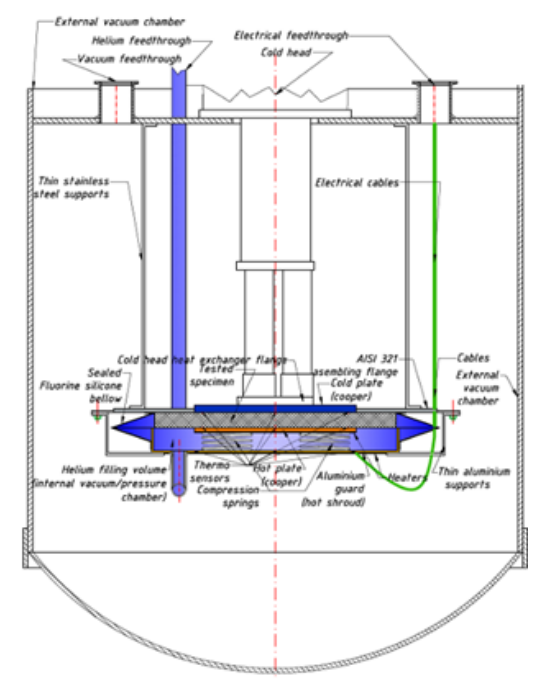

Fig. 2. Part of the FSU facility-like design model

After obtaining the geometric characteristics of the facility cryostat and experimental chamber, proceeding from the fact that in vacuum the main mechanism of heat transfer is radiation, the radiation power $Q_{r}, \mathrm{~W}$, between closed surfaces of bodies with temperatures $T_{h}$ (temperature of the "hot" surface) and $T_{c}$ (temperature of the cold surface), is determined in accordance with the Stefan - Boltzmann law for the case of radiant heat transfer between two gray surfaces in an enclosed space:

$$
Q_{r}=10^{8} \sigma \varepsilon_{m} A_{h}\left[\left(\frac{T_{h}}{100}\right)^{4}-\left(\frac{T_{c}}{100}\right)^{4}\right],
$$

where $\sigma$-Stefan - Boltzmann (blackbody radiation) constant, $5.77 \cdot 10^{-8} \mathrm{~W} \cdot \mathrm{m}^{-1} \cdot \mathrm{K}^{-4}$; $\varepsilon_{m}-$ mutual emissivity factor;

$A_{h}-$ hot surface area, $\mathrm{m}^{2}$;

$$
\varepsilon_{m}=\frac{1}{\frac{1}{\varepsilon_{h}}+\frac{A_{h}}{A_{c}}\left(\frac{1}{\varepsilon_{c}}-1\right)},
$$

where $\varepsilon_{h}$ - hot surface emissivity;

$\varepsilon_{c}$ - cold surface emissivity;

$A_{c}$ - cold surface area, $\mathrm{m}^{2}$.

The emissivity of an object reflects the difference in the radiation power of an absolutely black body and a real object (gray body), the same area heated to a given temperature. The issues of calculating the reduced emissivity coefficients of re-emitting systems are considered in [12; 13].

The modeling results presented in Fig. 2 system showed that to cool down the measuring system of such facility to the required temperatures, the cooling power of about $82 \mathrm{~W}$ at $10 \mathrm{~K}$ is required not counting the compensation of the thermal power released by the heaters of the measuring tool and supplied to the tool through thermal bridges (wires, supports, etc.)

Only a cooler of similar capacity (cryocooler complete with a helium compressor, connecting pipelines, controller) costs more than 200000 USD, which does not fit into the project budget.

The statement of the problem showed that it is necessary to create a calculation method to take into account the many factors involved in this task. Based on the method, a model should be created that allows to determine the characteristics of the cryogenic system elements. The obtained analytical 
solutions for the thermal states of the heat exchange surfaces and the already created model were taken as a basis.

Analysis of the simulation results showed that in order to significantly reduce the required cooling power, it is necessary to reduce the flow of radiated from the vacuum chamber surface heat power, which means the creation of an intermediate liquid nitrogen cryogenic shroud or, possibly, cryostat.

The first lesson learned from the modeling was that for proper design it is needed to create a sufficiently accurate and flexible thermophysical model of the liquid nitrogen cryogenic system, which ensures cooling of the measurement instrument and cryocooler surrounding area up to $80 \mathrm{~K}$.

\section{Proposed liquid nitrogen cooling system calculation method and modeling approach}

Taking into account the basic design and calculations, a calculation method and a flexible mathematical model of a cryogenic system were created and worked out, which made it possible to quickly take into account changes in thermophysical characteristics associated with the adoption of design decisions that ensured the creation of such a complex vacuum-cryogenic system.

The method was based on the concept of successive thermal resistances, through which the ambient heat is transferred to the thermal conductivity measuring instrument: passing through the wall of the outer vacuum chamber, heat is transferred from the inner wall surface to the cryostat shell by radiation and thermal conductivity through thermal bridges. From the inner surface of the cryostat shell heat is transferred to the refrigerant flow - liquid nitrogen. The inner shell of the cryostat, heated by a flow of liquid nitrogen, is a thermal energy irradiation source in relation to the pressure chamber and a thermal bridge to the cold head of the helium cryocooler.

The problems to be solved are to determine a sufficient liquid nitrogen flow rate and such a distance between the walls of the cryostat, at which the inner wall temperature is minimal.

After determining the geometric characteristics of the model elements, the temperature of the vacuum chamber wall inner surface is determined, which (like other elements of the model, where the thermal conductivity is calculated) is calculated for steady state conditions from the Fourier law:

$$
q=-k \nabla T,
$$

where $q$-vector of a local heat flux density, $\mathrm{W} \cdot \mathrm{m}^{-2}$;

$k$ - material thermal conductivity, $\mathrm{W} \cdot \mathrm{m}^{-1} \cdot \mathrm{K}^{-1}$;

$\nabla T$ - vector of a temperature gradient, $\mathrm{K} \cdot \mathrm{m}^{-1}$.

Further, according to (2-3) the heat flux of radiation to the wall of the cryostat is determined, and proceeding from (4), the heat fluxes along the thermal bridge are calculated. The temperature of the inner surface of the cryostat wall is determined. After that, the condition of temperature equilibrium on the surface of the inner wall of the cryostat is determined $T_{s}, \mathrm{~K}$ :

$$
T_{s}=T_{r e f}+\Delta T_{w},
$$

where $T_{\text {ref }}$ - refrigerant temperature, $\mathrm{K}$;

$\Delta T_{w}$ - temperature increase during convection heat transfer from the refrigerant to the cryostat wall, $\mathrm{K}$.

$\Delta T_{w}$ is determined by methods of the theory of hydraulic similarity, as:

$$
\Delta T_{w}=\frac{Q}{\pi r_{p} \alpha},
$$

where $Q-$ total heat flow on the cryostat inner surface, W;

$r_{p}$ - radius of the cryostat, $\mathrm{m}$;

$\alpha$ - heat transfer coefficient, $\mathrm{W} \cdot \mathrm{m}^{-2} \cdot \mathrm{K}^{-1}$.

The heat transfer coefficient $\alpha$ is determined based on the calculation of the Nusselt number, $\mathrm{Nu}$, obtained by calculating the flow conditions of the refrigerant:

$$
\alpha=\frac{N u \lambda_{r e f}}{d},
$$

where $\lambda_{\text {ref }}$ - liquid nitrogen thermal conductivity, $\mathrm{W} \cdot \mathrm{m}^{-1} \cdot \mathrm{K}^{-1}$; 


$$
d=2 r_{p} .
$$

The Nusselt number is determined by solving the Dittus-Boelter equation [15], for conditions of a flow of a single-phase heated liquid nitrogen:

$$
N u=0.023 \operatorname{Re}^{0.8} \operatorname{Pr}^{0.4}
$$

where: $R e$ - Reynolds number;

$\operatorname{Pr}-$ Prandtl number.

After determining the dimensionless hydraulic coefficients, the required nitrogen flow rate through the cryostat and the nitrogen mass flow rate are determined. Further, the hydraulic resistances are determined and the required nitrogen pressure at the inlet to the cryogenic system is determined, for which the Bernouli equation is solved:

$$
H_{\Sigma}=h+\frac{P}{\rho g}+\sum_{i=1}^{n} \alpha_{L i} \frac{V_{r e f i}^{2}}{2 g}+\sum_{j=1}^{n} \alpha_{R i} \frac{V_{r e f j}^{2}}{2 g},
$$

where $h$-liquid nitrogen column elevation, $\mathrm{m}$;

$P$-refrigerant residual pressure at the cryostat outlet, $\mathrm{Pa}$ (determined by maximal permitted liquid nitrogen temperature;

$\rho$ - density of the refrigerant, $\mathrm{kg} \cdot \mathrm{m}^{-3}$;

$\alpha_{L i}-$ dynamic head losses along the length of the pipes for $i$-system segment;

$V_{\text {refi }}$ - refrigerant velocity for $i$ - system segment;

$\alpha_{R j}$-dynamic head losses coefficients in local resistances for $j$-system local resistance;

$V_{\text {refj }}$ - refrigerant velocity for $j$ - system local resistance;

$g$ - acceleration of gravity at sea level, $9.80665 \mathrm{~m} \cdot \mathrm{s}^{-2}$.

Further, based on (2) and (3), the irradiation heat flux to the surface of the pressure chamber is determined. Then the radiation heat flux from the inner surface of the pressure chamber to the instrument for measuring thermal conductivity is calculated. Then the equation of the heat balance of the cooled plate of the thermal conductivity measuring instrument is solved and, based on the required temperature range $(14-20 \mathrm{~K})$, the required cooling capacity of the cryocooler is determined. The complete diagram of the computational model obtained on the basis of the proposed method is shown in Fig. 4.

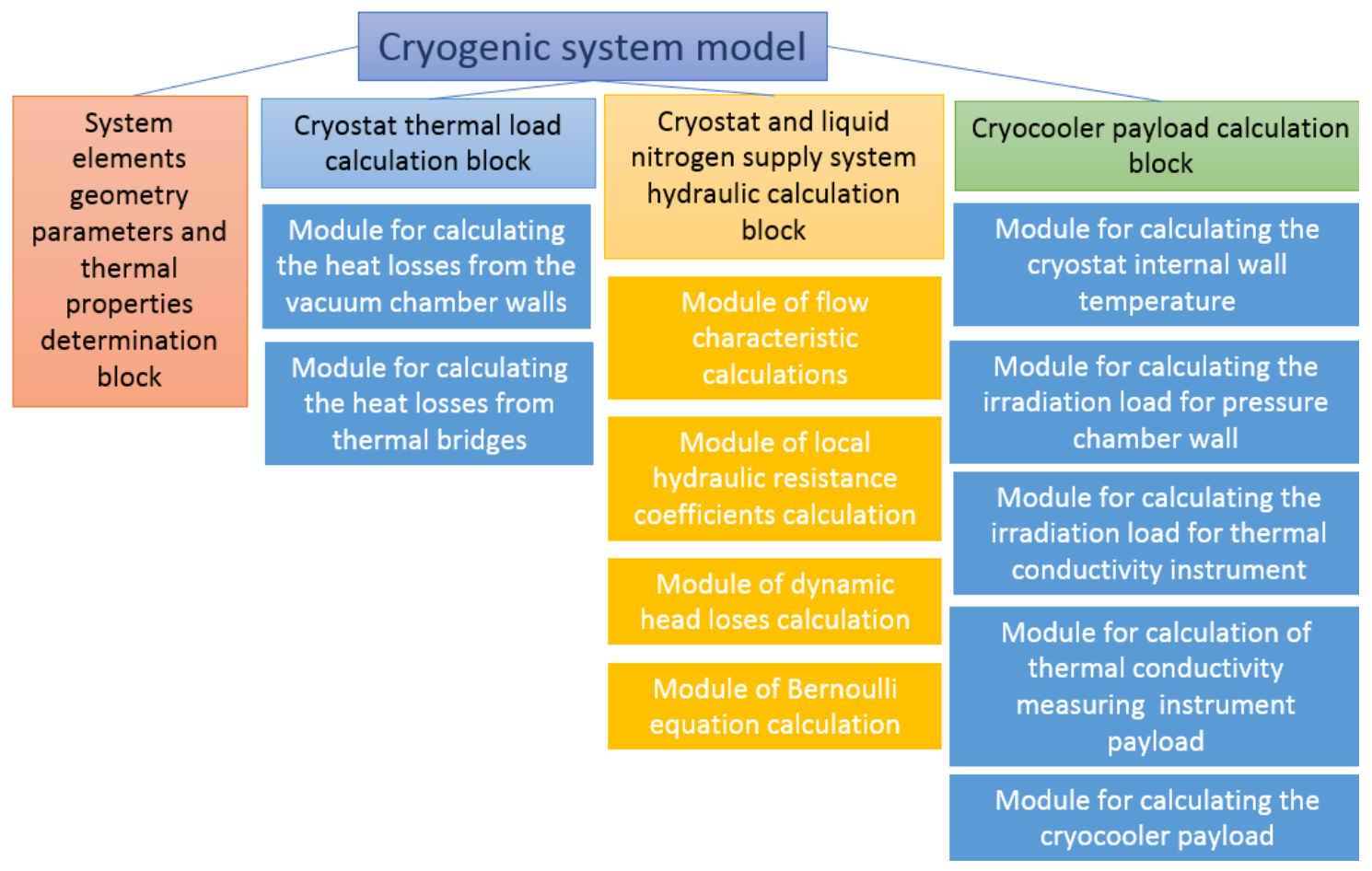

Fig. 4. Cryogenic system model diagram 
The main constrain for the system modelling is that budget models of cryocoolers have a refrigeration power limit of less than $15 \mathrm{~W}$ at $4 \mathrm{~K}$.

\section{Results of modelling and consequent facility design improvement}

As a result of the simulation, the dependence of the cryostat wall temperature on the flow rate of liquid nitrogen through the cryostat at a calculated heat load was determined. The result of modelling is shown in Fig. 5.

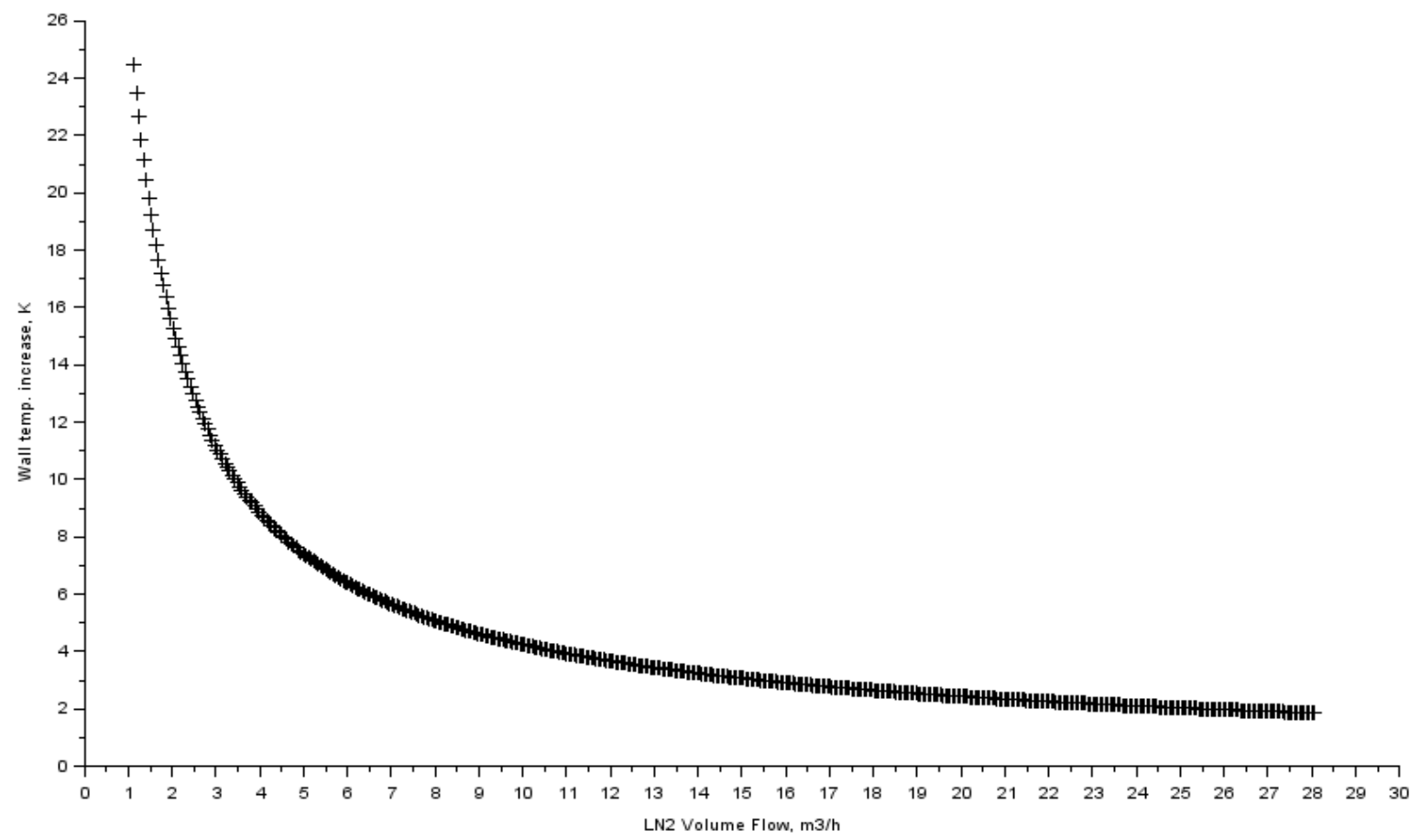

Fig. 5. Needed liquid nitrogen volume flow rate in cryostat

It is necessary to provide a mode of weak wall boiling in the cryostat, then its efficiency will be close to the calculated one. According to the table of vapour-liquid content of liquid nitrogen at a pressure close to atmospheric (101.3 $\mathrm{kPa}$ with free boiling conditions in a cryostat), the maximum permissible temperature rise of the cryostat wall will be about $5.5 \mathrm{~K}$, but it is desirable that it does not exceed 2.3-2.4 K. It means, that minimum permissible liquid nitrogen volume flow rate is $7.2944 \mathrm{~m}^{3} \cdot \mathrm{h}^{-1}$ (the wall temperature increasing will be $5.5038 \mathrm{~K}$ ), and the optimum is $20.4242 \mathrm{~m}^{3} \cdot \mathrm{h}^{-1}$.

Also, the optimum geometry of the cryostat was determined: the outer cylinder diameter is $600 \mathrm{~mm}$, the inner cylinder diameter is $500 \mathrm{~mm}$, and the minimum distance between the walls of the cryostat, at which the effect of the temperature of the outer wall of the cryostat on the temperature of the inner wall is minimal at a given flow rate is $40 \mathrm{~mm}$.

Then the minimum mass flow rate of liquid nitrogen through the cryostat was determined. With a safety factor of $15 \%$, the flow rate of liquid nitrogen of $6384 \mathrm{~kg}$ per hour was taken as the value that determines the parameters of the pump and storage system.

Based on the results of modelling and analysis, it was decided to place the thermal conductivity measuring instrument itself in a sealed pressure chamber, into which helium can be injected and the pressure in which can be adjusted from high vacuum to 1.5-2 bar. This pressure chamber should be in a vacuum inside the nitrogen cryostat and have as few thermal bridges as possible with it. The measuring instrument located in the pressure chamber must be connected by a thermal bridge with a helium cryocooler, which provides cooling of the cold plate of the measuring instrument.

The nitrogen cryostat must be made in the form of an optically opaque vessel, mainly consisting of a volumetric vessel that is filled with liquid nitrogen, which has an internal cavity that communicates with the external vacuum volume and in which a pressure chamber with a measuring instrument is mounted. The cryostat is mounted in a high-vacuum vessel equipped with intermediate reflective surfaces that provide effective high-vacuum thermal insulation. 
Based on the modelling results, the following layout diagram was adopted, Fig. 6, which became the basis for the development of the facility design documentation.

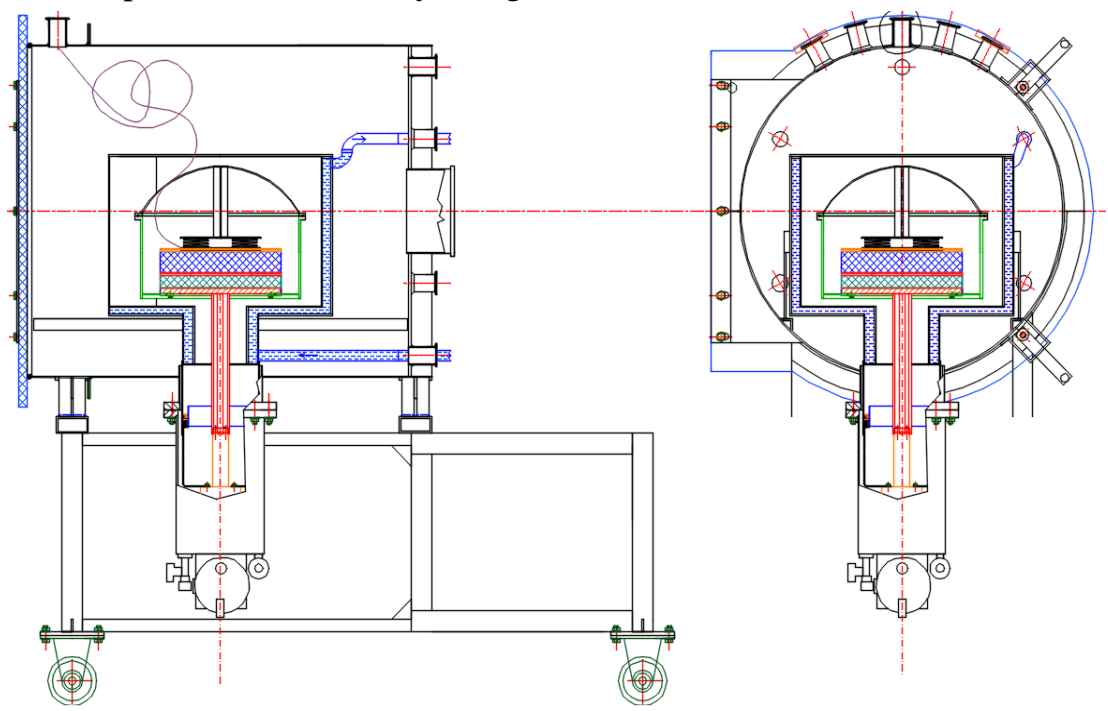

Fig. 6. Final modelling layout

On the basis of this layout and the simulation results, the design documentation was developed, and the facility is being manufactured. As a result, the principle of an intermediate cryostat with liquid nitrogen was implemented, despite the enormous complexity of assembling many mutually mating surfaces and parts, Fig. 7.

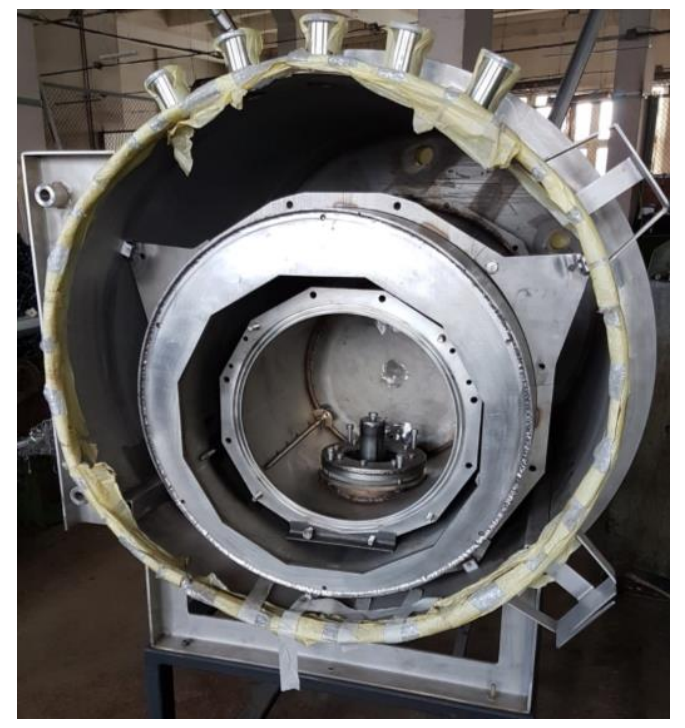

Fig. 7. Thermal conductivity measuring facility actual development

The facility is currently under assembling and integration processes. It is planned to complete the assembly, integration and intermediate tests of individual parts and systems of the facility in May-June 2021, after which it is planned to start full-scale testing and verification. The verification of the facility is planned as a metrological system for measuring low temperature thermal conductivity.

\section{Conclusions}

Despite the fact that tests of the thermal conductivity measurement facility have not yet been carried out, at this stage we can draw the following conclusions:

1. The developed under proposed calculation methodology model made it possible to quickly update the calculations after making the necessary changes in the design of the facility according to the conditions of assembling or other technology requirements and, in turn, to verify the changes made, or force additional changes to keep the original technical requirements for the cryocooler; 
2. The application of the proposed flexible method and model made it possible not only to overcome the limitations associated with the impossibility of using a high-power helium refrigeration machine, but also to provide an operational control calculation of the cryogenic system parameters, when changes were made to the design for technological reasons, which made it possible to manufacture this complex and precision measuring instrument;

3. The proposed approach will solve the problem of measuring the thermal conductivity of polyurethane thermal insulation in the operating conditions, required for the space industry.

In the course of the facility testing and verification, the proposed methodology and the model developed on its basis will also be finally verified, but already now we can talk about its sufficient flexibility for the design and calculation purposes. The proposed method, model and approach can be useful for companies and research institutes creating new types of cryogenic thermal insulation and low temperature test equipment.

\section{Acknowledgments}

The authors are grateful for the support of the Competence Center of Electrical and Optical Equipment Production Sector of Latvia, The Central Finance and Contracting Agency (CFCA) of Latvia, and the European Regional Development Fund, within the framework of which Project No. 1.2.1.1/18/A/006 Research no. 2.8. "Cryogenic Insulation Thermal Conductivity Testing System", the discussed facility is designed and manufactured.

\section{References}

[1] Federal Aviation Administration. Space Operations. 4.2.1. Rockets and Launch Vehicles. Washington: FAA, 2021.

[2] United Launch Alliance. Atlas V launch services user's guide. Revision 11. Centennial: United Launch Alliance, 2010. 420 p.

[3] Scholtens B.E., Fesmire J.E., Sass J.P., Augustynowicz S.D., Heckle, K.W. Cryogenic thermal performance testing of bulk-fill and aerogel insulation materials. Transactions of the American Institute of Physics Cryogenic Engineering Conference, 985, 152 (2008) - CEC, Vol. 53, March 27, 2008, College Park, Maryland, USA, p.p. 152-159.

[4] Fesmire J.E., Sass J. Aerogel insulation applications for liquid hydrogen launch vehicle tanks. Cryogenics, Volume 48, Issues 5-6, May-June 2008, pp. 223-231.

[5] Powell R.L., Childs G.E. Thermal Conductivity. American Institute of Physics Handbook. Section 4g. New York: McGraw-Hill, 1972. 2368 p.

[6] Darkow N., Fischer A., Scheufler H., Hellmann H, Gerstmann J. Concept development of a cryogenic tank insulation for reusable launch vehicle. Proceedings of 8th European conference for aeronautics and space sciences (EUCASS), July 1-4, 2019, Madrid, Spain, pp. 1-11.

[7] Golyand M. Calculations and tests of thermal insulation. Moscow: Gostoptekhizdat, 1961. 316 p.

[8] Scott R.B. Cryogenic Engineering. New York: D.Van Nostrand Company Inc., 1962. 386 p.

[9] Hafer R.A. Cryovacuum technology. Heidelberg: Springer, 1981.272p.

[10] ASTM C177-19 standard "Test Method for Steady-State Heat Flux Measurements and Thermal Transmission Properties by Means of the Guarded-Hot-Plate Apparatus".

[11] Miller R.A., Kuczmarski M.A. Method for Measuring Thermal Conductivity of Small Samples Having Very Low Thermal Conductivity. NASA/TM-2009-215460. Cleveland: NASA Glenn Research Center, 2009. 31p.

[12] EN 12667:2001 standard "Thermal performance of building materials and products - Determination of thermal resistance by means of guarded hot plate and heat flow meter methods - Products of high and medium thermal resistance".

[13] ISO 13787:2003 standard "Thermal insulation products for building equipment and industrial installations - Determination of declared thermal conductivity".

[14] Barrios M. Material Characterization of Rigid Foam Insulation at Low Temperature. Thesis (Ph.D.) Tallahasse: Florida State University, College of Engineering, 2011. 106 p.

[15] Incopera F.R. Fundamentals of Heat and Mass Transfere. New York: John Willey \& Sons, 2011. $1070 \mathrm{p}$. 